\title{
Thoracoscopic Enucleation of Esophageal Submucosal Tumor
}

\section{Wu MH* and Wu HY}

Department of Surgery, Tainan Municipal Hospital, Taiwan

*Corresponding author: Ming-Ho Wu, Department of Surgery, Tainan Municipal Hospital, Taiwan

Received: January 19, 2017; Accepted: February 17, 2017; Published: February 22, 2017

\begin{abstract}
Preoperative diagnosis and surgical approach are critical points in the management of posterior mediastinal lesions. A 77 year-old woman with esophageal submucosal tumor underwent thoracoscopic enucleation of the tumor. Clinically, she presented with dysphagia and epigastric discomfort. The patient was uneventful discharged on postoperative day 3. Postoperative esophagography showed normal patency. Perioperative images are interest.
\end{abstract}

Keywords: Esophageal submucosal tumor; Thoracoscopic excision; Mediastinal bronchogenic cyst

\section{Introduction}

Esophageal submucosal tumor could locate at any area of whole course of the esophagus [1]. The disease usually present with dysphagia that resulted from tumor compression of the esophagus. Here, we present a patient with a $3.6 \mathrm{~cm}$ esophageal submucosal tumor. The patient underwent thoracoscopic enucleation of the tumor. We will focus on the thoracoscopic enucleation and perioperative images.

\section{Case Presentation}

A 77-year-old woman complained of dysphagia and epigastric discomfort for more than 3 months. Chest film showed normal airways. Esophagography showed submucosal defect at lower thoracic esophagus (Figure 1). Endoscopy revealed a submucosal lesion located at lower thoracic esophagus (Figure 2). Contrast computed tomography revealed a $3.6 \mathrm{~cm}$ heterogeneous density bulging mass at the lower esophagus (Figure 3). The surgical procedure was as following; a $4 \mathrm{~cm}$ incision was made at left $5^{\text {th }}$ intercostal space of middle axillary line and a port at left $9^{\text {th }}$ intercostal space of posterior axillary line. A $3.6 \mathrm{~cm}$ lesion in the lower third esophageal submucosa was carefully dissected free from the adjacent tissue (Figure 4). Operation time was 60 minutes. Histology was proved to be

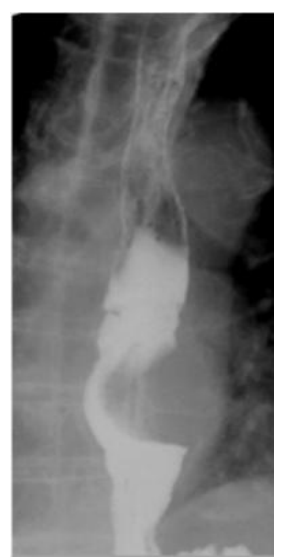

Figure 1: Esophagography showed a submucosal defect at lower thoracic esophagus.

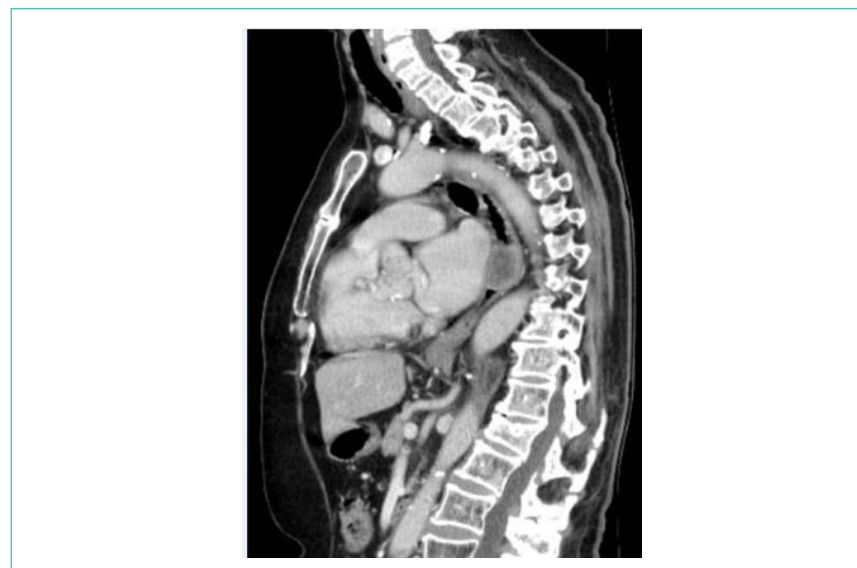

Figure 2: Chest computed tomography showed a heterogenous density mass at lower thoracic esophagus.

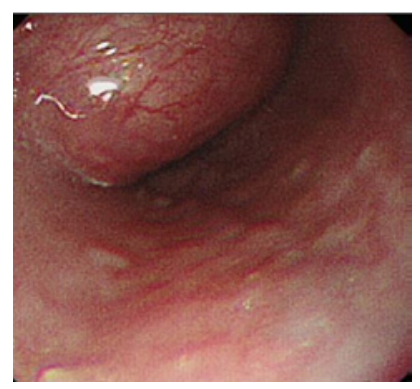

Figure 3: Endoscopic finding showed an esophageal submucosal lesion.

esophageal submucosal gastrointestinal stromal tumor. Postoperative esophagography showed normal patency (Figure 5).

\section{Comments}

Different imaging techniques can be used for preoperative diagnosis and differential diagnoses included a duplication cyst, esophageal leiomyoma, pleural fibroma, lymphadenopathy and bronchogenic cyst. Despite improved imaging modalities, a definitive diagnosis remains difficult preoperatively [1]. In the presenting case, esophageal submucosal tumor was diagnosed before surgical
Austin J Surg - Volume 4 Issue 1 - 2017

ISSN : 2381-9030 | www.austinpublishing group.com

Wu et al. (C) All rights are reserved
Citation: Wu MH and Wu HY. Thoracoscopic Enucleation of Esophageal Submucosal Tumor. Austin J Surg. 2017; 4(1): 1096 


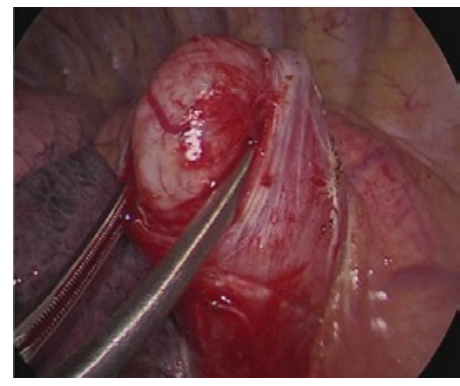

Figure 4: Thoracoscopic findings showed the esophageal submucosal tumor.

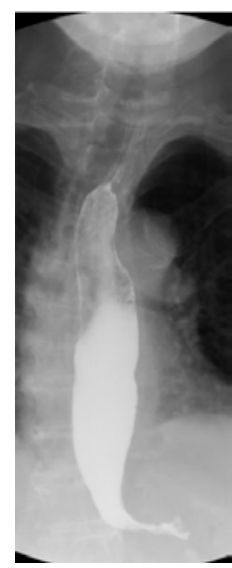

Figure 5: Postoperative esophagography showed normal esophagus.

intervention. Esophageal submucosal tumors originating from the muscularis propria layer could be treated using submucosal tunneling endoscopic resection with a high successful rate, even when the size of the tumor was larger than $35 \mathrm{~mm}$ [2]. The above endoscopic procedure had occasional complication that required further treatment [3]. A giant esophageal submucosal tumor has been reported, which required esophagectomy [4]. Thoracoscopic excision of esophageal submucosal tumor was feasible [5]. In our presenting case of esophageal submucosal tumor, thoracoscopic enucleation was very useful because the $3.6 \mathrm{~cm}$ tumor was easily identified and excised without difficulty.

\section{References}

1. McAdams HP, Kirejczyk WM, Rosado-de-Christenson ML, Matsumoto S. Bronchogenic cyst: Imaging features with clinical and histopathologic correlation. Radiology. 2000; 217: 441-446.

2. Wang $\mathrm{H}$, Tan $\mathrm{Y}$, Zhou $\mathrm{Y}$, Wang $\mathrm{Y}$, Li C, Zhou J, et al. Submucosal tunneling endoscopic resection for upper gastrointestinal submucosal tumors originating from the muscularis propria layer. Eur J Gastroenterol Hepatol. 2015; $27: 776-780$

3. Chen T, Zhang C, Yao LQ, Zhou PH, Zhong YS, Zhang YQ, et al. Management of the complications of submucosal tunneling endoscopic resection for upper gastrointestinal submucosal tumors. Endoscopy. 2016; 48: 149-155.

4. Nakano A, Akutsu $\mathrm{Y}$, Shuto $\mathrm{K}$, Uesato M, Kono T, Hoshino I, et al. Giant esophageal gastrointestinal stromal tumor: report of a case. Surg Today. 2015; 45: 247-252.

5. Watanabe T, Miyazaki T, Saito H, Yoshida T, Kumakura Y, Honjyo H, et al. Resection of an esophageal schwannoma with thoracoscopic surgery: a case report. Surg Case Rep. 2016; 2: 127.
Austin J Surg - Volume 4 Issue 1 - 2017

ISSN : 2381-9030 | www.austinpublishing group.com Wu et al. (C) All rights are reserved
Citation: Wu MH and Wu HY. Thoracoscopic Enucleation of Esophageal Submucosal Tumor. Austin J Surg. 2017; 4(1): 1096. 